\title{
Differentiation between Benign and Malignant Solid Thyroid Nodules Using an US Classification System
}

\author{
Young Hun Lee, $M D^{1}$, Dong Wook Kim, $M D^{1}$, Hyun Sin In, $M D^{1}$, Ji Sung Park, $M D^{1}$, Sang Hyo Kim, $M D^{2}$, \\ Jae Wook Eom, $M D^{3}$, Bomi Kim, $M D^{4}$, Eun Joo Lee, $M D^{5}$, Myung Ho Rho, $M D^{6}$ \\ Departments of ${ }^{1}$ Radiology, ${ }^{2}$ General Surgery (Thyroid \& Breast Clinic), ${ }^{3}$ Otorhinolaryngology-Head \& Neck Surgery, and ${ }^{4}$ Pathology, Busan Paik \\ Hospital, Inje University College of Medicine, Busan 614-725, Korea; ${ }^{5}$ Department of Radiology, Cancer Center, Dongnam Institute of Radiological \\ and Medical Science, Busan 619-953, Korea; ${ }^{6}$ Department of Radiology, Kangbuk Samsung Hospital, Sungkyunkwan University School of Medicine, \\ Seoul 110-746, Korea
}

Objective: To evaluate the diagnostic accuracy of a new ultrasound (US) classification system for differentiating between benign and malignant solid thyroid nodules.

Materials and Methods: In this study, we enrolled 191 consecutive patients who received real-time US and subsequent US diagnoses for solid thyroid nodules, and underwent US-guided fine-needle aspiration. Each thyroid nodule was prospectively classified into 1 of 5 diagnostic categories by real-time US: "malignant," "suspicious for malignancy," "borderline," "probably benign," and "benign". We evaluated the diagnostic accuracy of thyroid US and the cut-off US criteria by comparing the US diagnoses of thyroid nodules with cytopathologic results.

Results: Of the 191 solid nodules, 103 were subjected to thyroid surgery. US categories for these 191 nodules were malignant $(n=52)$, suspicious for malignancy $(n=16)$, borderline $(n=23)$, probably benign $(n=18)$, and benign $(n=$ 82). A receiver-operating characteristic curve analysis revealed that the US diagnosis for solid thyroid nodules using the 5 -category US classification system was very good. The sensitivity, specificity, positive and negative predictive values, and accuracy of US diagnosis were $86 \%, 95 \%, 91 \%, 92 \%$, and $92 \%$, respectively, when benign, probably benign, and borderline categories were collectively classified as benign (negative).

Conclusion: The diagnostic accuracy of thyroid US for solid thyroid nodules is high when the above-mentioned US classification system is applied.

Index terms: Thyroid nodule; Solid, Ultrasound; Fine-needle aspiration; Classification; Malignancy

Received June 15, 2010; accepted after revision April 4, 2011. This work was supported by Grant from Inje University, 2009. Corresponding author: Dong Wook Kim, MD, Department of Radiology, Busan Paik Hospital, Inje University College of Medicine, 633-165 Gaegeum-dong, Busanjin-gu, Busan 614-725, Korea.

- Tel: (8251) 890-6549 - Fax: (8251) 896-1085

- E-mail: dwultra@lycos.co.kr

This is an 0pen Access article distributed under the terms of the Creative Commons Attribution Non-Commercial License (http://creativecommons.org/licenses/by-nc/3.0) which permits unrestricted non-commercial use, distribution, and reproduction in any medium, provided the original work is properly cited.

\section{INTRODUCTION}

Thyroid ultrasound (US) is the major diagnostic modality for evaluating thyroid nodules. Using US, a thyroid nodule appears as a nodular lesion within the thyroid gland that is distinguishable from the adjacent parenchyma. Several US features, such as marked hypoechogenicity, irregular margin, microcalcifications, and a taller-than-wide shape have been introduced as potential predictors for the presence of thyroid malignancies (1-10). However, many studies report variability in the diagnostic accuracy or 
a considerable overlap in the appearance for distinction between benign and malignant thyroid nodules, regardless of whether it has a solid or cystic US configuration (1-14). This overlap of benign and malignant thyroid nodules in US diagnosis may be related to the fact that previous studies which used a simple US diagnostic category $(1,5,7,11$, 13), did not differentiate between solid and cystic thyroid nodules $(2,3,5,8-14)$, and further, employed retrospective image analyses $(6-12,14)$. Recently, several investigators have suggested that malignant US features of partially cystic thyroid nodules (PCTNs) are considerably different from those of solid nodules and include an eccentric configuration with an acute angle, microcalcifications within solid component, macrolobulated or irregular freemargin of the solid component, perinodular infiltration, and a centripetal vascularity in the pedicle $(15,16)$.

A few prospective studies report the diagnostic accuracy of thyroid US for thyroid nodules (1-4). In particular, Horvath et al. (4) proposed the Thyroid Imaging Reporting and Data System (TIRADS) to develop a standardized US characterization and data reporting system for thyroid lesions. However, to the best of our knowledge, there are no existing studies that simultaneously utilize the prospective real-time US classification for solid thyroid nodules.

The aim of this study is to assess the accuracy of US diagnosis for benign and malignant solid thyroid nodules using a real-time US performance and classification system with 5 categories.

\section{MATERIALS AND METHODS}

\section{Study Population}

From January 2008 to June 2008, one radiologist performed thyroid US and made prospective US diagnoses for solid thyroid nodules in 726 patients ( $M: F=114: 612$; mean age, 51.1 [14.3] years). Among them, 195 patients with solid thyroid nodules with a maximum diameter $>5$ $\mathrm{mm}$ and cytologically diagnosed by a consecutive US-guided fine-needle aspiration (US-FNA) following the initial thyroid US, were enrolled in this study. A solid thyroid nodule was defined as purely solid or predominantly solid with a cystic component comprising less than $10 \%$ of the total volume. of the 195 patients examined, four nodules were excluded because their nodules were diagnosed as benign lesions based on clinical and sonographic information such as a history of previous ethanol injection, spontaneous collapse, or FNA for benign cystic thyroid nodules. Five hundred thirty-one patients who only underwent real-time thyroid US without subsequent US-FNA or thyroid surgery were excluded from the study. The cytopathological results of solid thyroid nodules in 191 patients were the diagnostic reference standard for real-time US diagnosis. In the cases of multiple thyroid nodules, only one nodule was selected for each patient in US-FNA on the basis of the highest likelihood of malignancy, solidity, and the largest diameter. We obtained written informed consent from all patients prior to each US-FNA. Our Institutional Review Board approved this study.

\section{Thyroid US}

Thyroid US was performed by a radiologist with a highresolution ultrasound instrument (iU 22; Phillips Medical Systems, Bothell, WA) equipped with a 12-15 MHz linear probe. As depicted in the thyroid US, each thyroid nodule was classified into 1 of 5 categories immediately after realtime US examination by the same radiologist. The categories included "malignant," "suspicious for malignancy," "borderline," "probably benign," and "benign" (Fig. 1).

The inclusion criteria for the sonographic features of solid thyroid nodules were as follows: 1 ) US characteristics of malignancy including marked hypoechogenicity, a margin related to thyroid malignancy, microcalcifications, a tallerthan-wide shape, and associated lymphadenopathy with intranodal cystic components or microcalcifications in the perithyroidal area (Fig. 2). Marked hypoechogenicity was defined as having the same or decreased echogenicity compared with the strap muscle. A margin related to thyroid malignancy included microlobulation, a spiculated margin, and perithyroidal infiltration. Microlobulation was defined as having the presence of many small lobules on the surface of a nodule; a spiculated margin was defined as having the presence of irregular spiculation on the surface of a nodule; perithyroidal infiltration was defined as having the extension of the lesion into an extracapsular area. Microcalcifications were defined as tiny, hyperechoic foci ( $<1 \mathrm{~mm}$ in size) with no comet-tail artifacts or posterior shadow. A taller-than-wide shape was defined as a nodule having a greater anteroposterior diameter than its transverse diameter. 2) The US characteristics of a benign nodule included an ovoid or flat shape, isoechogenicity, a smooth margin, and peripheral vascularity. Peripheral vascularity was defined as vascular predominance in the periphery of the nodule in a color Doppler US. Color Doppler US with a low pulse repetition frequency $(700 \mathrm{~Hz})$ was 


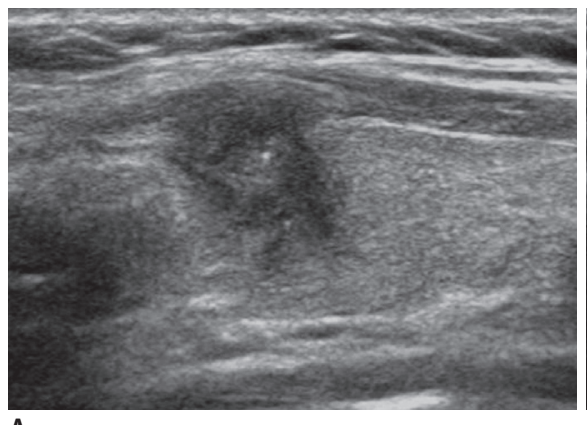

A

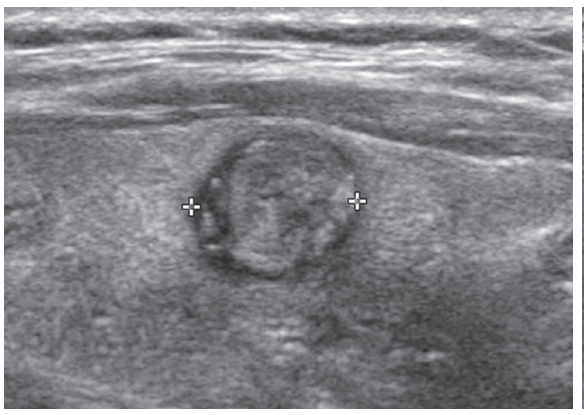

D
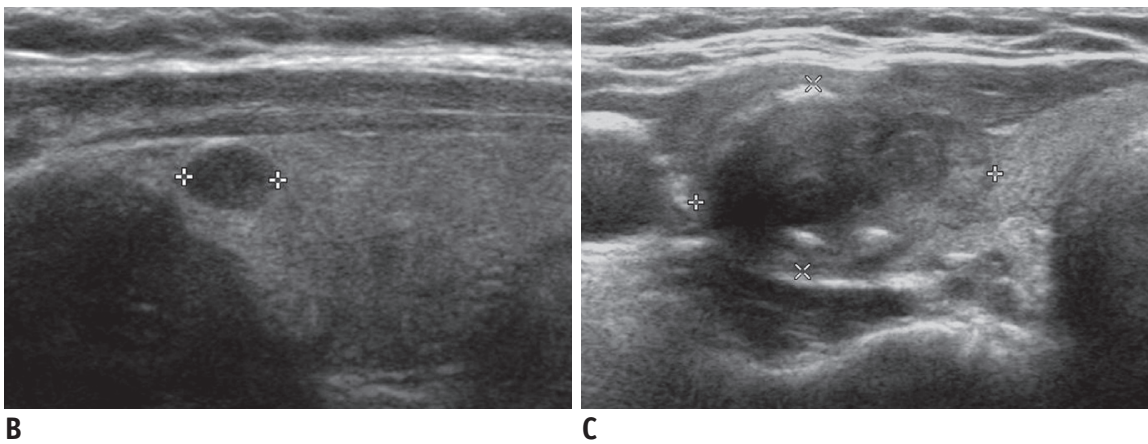

C

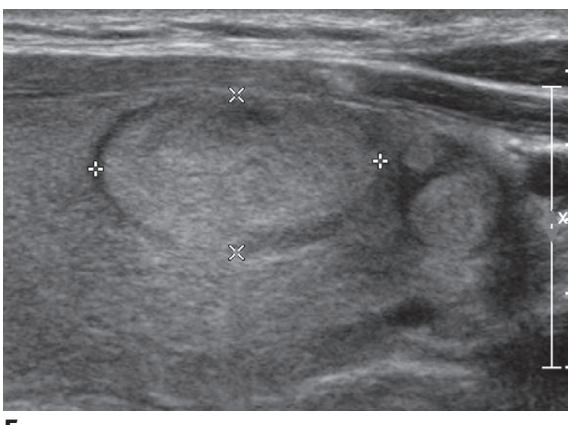

$\mathbf{E}$

Fig. 1. Five categories for US diagnosis of solid thyroid nodules.

A. Malignant. Longitudinal US image of papillary thyroid carcinoma in 42-year-old woman shows marked hypoechogenicity, spiculated margin, microcalcifications, and taller-than-wide shape for nodule. B. Suspicious for malignancy. Longitudinal US image of papillary thyroid carcinoma in 42-year-old woman shows marked hypoechogenicity, smooth margin, and ovoid shape. C. Borderline. Transverse US image of nodular hyperplasia in 60-year-old woman shows macrocalcification in peripheral portion of nodule. Patient underwent right lobectomy of thyroid, despite benign cytology upon US-guided fine-needle aspiration, for pathologic confirmation. D. Probably benign. Longitudinal US image of benign nodule in 57-year-old woman shows isoechogenicity and smooth margin. E. Benign. Longitudinal US image of benign nodule in 46-year-old woman shows ovoid shape, isoechogenicity, and smooth margin.

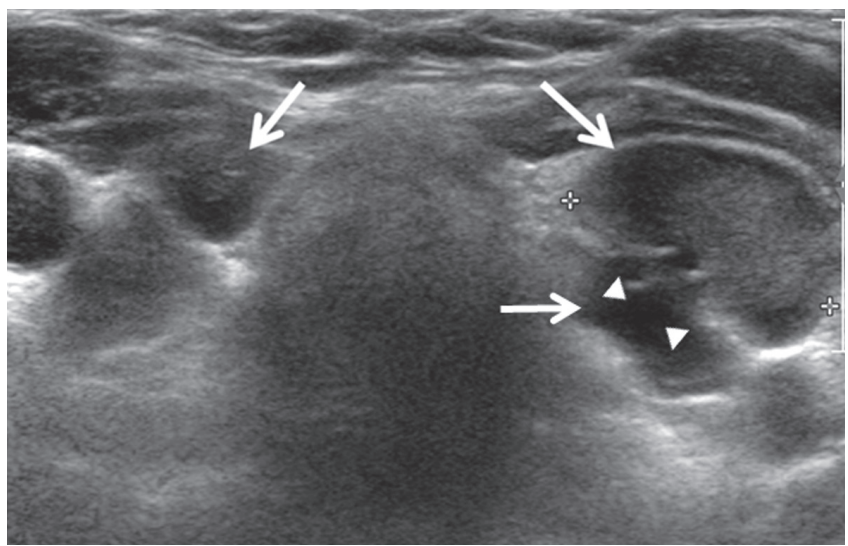

Fig. 2. Metastatic lymphadenopathy of papillary thyroid carcinoma in perithyroidal region. Transverse US image in 27-yearold woman shows associated lymph nodes (arrows) with intranodal cystic components (arrowheads) along both tracheoesophageal grooves in level of upper trachea.

used to evaluate the vascularity of a thyroid nodule. 3 ) Borderline US characteristics of a thyroid nodule included hypoechogenicity, centrally predominant vascularity, and macrocalcifications, including egg shell calcification and intranodular macrocalcifications. Hypoechogenicity was defined as increased and decreased echogenicity compared with the strap muscle and the adjacent thyroid parenchyma, respectively.

\section{Nodule Classification}

The criteria for the US diagnosis of a thyroid nodule included the following: if a thyroid nodule exhibited two or more US characteristics that suggested malignancy in the thyroid US regardless of the existence of borderline or benign US features, it was considered malignant; if a thyroid nodule exhibited one US characteristic for malignancy in the thyroid US regardless of the existence of borderline or benign US features, it was considered as being suspicious for malignancy; if a thyroid nodule exhibited one or more borderline US features without US characteristics that suggest malignancy regardless of the existence of benign US features, it was considered to be borderline; if a thyroid nodule exhibited one or two US characteristics that suggest a benign nodule with no malignancy or borderline US features, it was considered as being probably benign; if a thyroid nodule exhibited three or more US characteristics 
of a benign nodule with no malignancy or borderline US features, it was considered to be benign. All thyroid nodules were assessed by a single radiologist using real-time thyroid US (Fig. 3).

\section{US-Guided Fine-Needle Aspiration and Reference}

Standard

US-guided fine-needle aspiration was performed once for each thyroid nodule without the administration of local anesthesia. For each sample, a smear was made on 4-6 slides that were fixed in 95\% ethanol and sent to the department of pathology for Papanicolaou staining. All 191 patients ( $M: F=28: 163$; mean age, 48.0 [12.4] years) with nodules $(n=191$; size range, $0.5-8.2 \mathrm{~cm}$; mean, 1.53 [1.1] $\mathrm{cm}$ ) underwent US-FNA for the following reasons: suspicious US diagnosis $(n=68)$, borderline US diagnosis $(n=23)$, screening $(n=77)$, insufficient cytology after palpationguided fine-needle aspiration biopsy $(n=12)$, positive risk factor for thyroid malignancy $(n=5)$, and patient request ( $n$ $=6$ ).

We compared US diagnoses with cytopathologic results. Thyroid nodules classified as suspicious for malignancy and malignant were collectively classified as malignant, and thyroid nodules classified as probably benign and benign were collectively classified as benign.

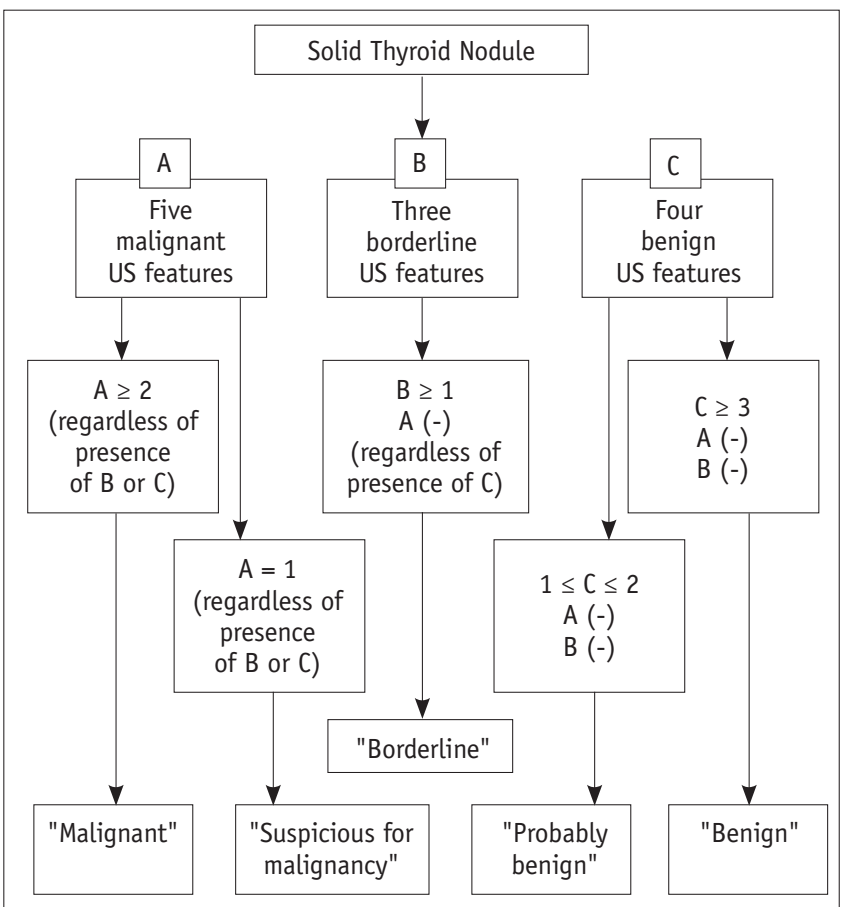

Fig. 3. US classification system with 5 categories for diagnosis of solid thyroid nodules.

\section{Statistical Analysis}

The sensitivity, specificity, positive and negative predictive values, and accuracy for the US diagnosis of thyroid nodules were calculated. The highest diagnostic accuracy of US diagnosis depending on classification as either benign or malignant using 5 US categories, was determined by the receiver-operating characteristic (ROC) analysis. To evaluate cut-off US criteria for thyroid malignancy and the ability of the 5-category system to allow differentiation between malignant and benign nodules, the areas under the ROC curve $(\mathrm{Az})$ were determined. Statistically significant differences between $\mathrm{Az}$ values are reported as $95 \%$ confidence intervals. Statistical analyses were performed with the SPSS software package for Windows (SPSS, Inc., Chicago, IL). Two-tailed $p$ values less than $5 \%$ were considered to be statistically significant.

\section{RESULTS}

After the thyroid US examination of 191 patients, one radiologist prospectively classified each thyroid nodule as follows: malignant ( $n=52)$, suspicious for malignancy ( $n$ $=16)$, borderline $(n=23)$, probably benign $(n=18)$, and benign ( $n=82)$. The US classifications with cytopathologic results in 191 thyroid nodules are summarized in Table 1.

Of these patients, $103(\mathrm{M}: \mathrm{F}=14: 89$; mean age, 48.0 years) underwent thyroid surgery due to malignant cytologic results $(n=67)$, positive US diagnosis despite benign cytologic results upon consecutive US-FNA $(n=$ $6)$, indeterminate cytology $(n=8)$, presence of a large palpable mass with a benign cytology $(n=14)$, and patient request $(n=8)$. Repeated US-FNA was performed on thyroid nodules when the cytological results of the first US-FNA were indeterminate for malignancy $(n=10)$ or inadequate $(n=5)$. The nodules $(n=81)$ assigned to the benign US category and benign cytology were followed up with US examination after at least 12 months; a significant interval change, such as new detection of suspicious US features or significant increase in nodular size $(50 \%$ or more increase in nodular volume) was not found. Out of the 191 patients, 43 exhibited a solitary thyroid nodule and 148 had two or more thyroid nodules.

In the ROC analysis, the diagnostic accuracy of US diagnosis was highest when malignant and suspicious for malignancy were classified as malignant and other US categories were classified as benign. The sensitivity, specificity, positive and negative predictive values, and 
accuracy of thyroid US for differentiating a malignant lesion from a benign one were $86 \%, 95 \%, 91 \%, 92 \%$, and $92 \%$, respectively. Diagnostic indices for individual US categories are shown in Table 2. The US categories for the diagnosis of malignant and benign resulted in a high predictive value;
$100 \%$ positive predictive value for malignant, $86 \%$ negative predictive value for malignant, and $96 \%$ positive predictive value for benign. However, the suspicious for malignancy US category yielded a relatively low diagnostic accuracy value.

The cut-off US criteria applied for thyroid malignancy

Table 1. Comparison of US Diagnoses and Cytopathological Results

\begin{tabular}{lccc}
\hline \multirow{2}{*}{ US Classifications } & \multicolumn{3}{c}{ Cytopathological Results } \\
\cline { 2 - 4 } & Malignant & Benign & Total \\
\hline Malignant & 52 & 0 & 52 \\
Suspicious for malignancy & 10 & 6 & 16 \\
Borderline & 4 & 19 & 23 \\
Probably benign & 3 & 15 & 18 \\
Benign & 3 & 79 & 82 \\
\hline Total & 72 & 119 & 191 \\
\hline
\end{tabular}

Table 2. Diagnostic Indices for Individual US Categories

\begin{tabular}{lcccrr}
\hline & Sensitivity (\%) & Specificity (\%) & PPV (\%) & NPV (\%) & Accuracy (\%) \\
\hline Malignant & $52 / 72(72)$ & $119 / 119(100)$ & $52 / 52(100)$ & $119 / 139(86)$ & $171 / 191(90)$ \\
Suspicious for malignancy & $10 / 72(14)$ & $113 / 119(95)$ & $10 / 16(63)$ & $113 / 175(65)$ & $123 / 191(64)$ \\
Borderline* $^{*}$ & $19 / 119(16)$ & $68 / 72(64)$ & $19 / 23(83)$ & $68 / 168(41)$ & $87 / 191(46)$ \\
Probably benign & $15 / 119(13)$ & $69 / 72(96)$ & $15 / 18(83)$ & $69 / 173(40)$ & $84 / 191(44)$ \\
Benign & $79 / 119(66)$ & $69 / 72(96)$ & $79 / 82(96)$ & $69 / 109(63)$ & $148 / 191(78)$ \\
\hline
\end{tabular}

Note. $-{ }^{*}$ Diagnostic index of borderline class when this was classified as benign. NPV = negative predictive value, PPV = positive predictive value

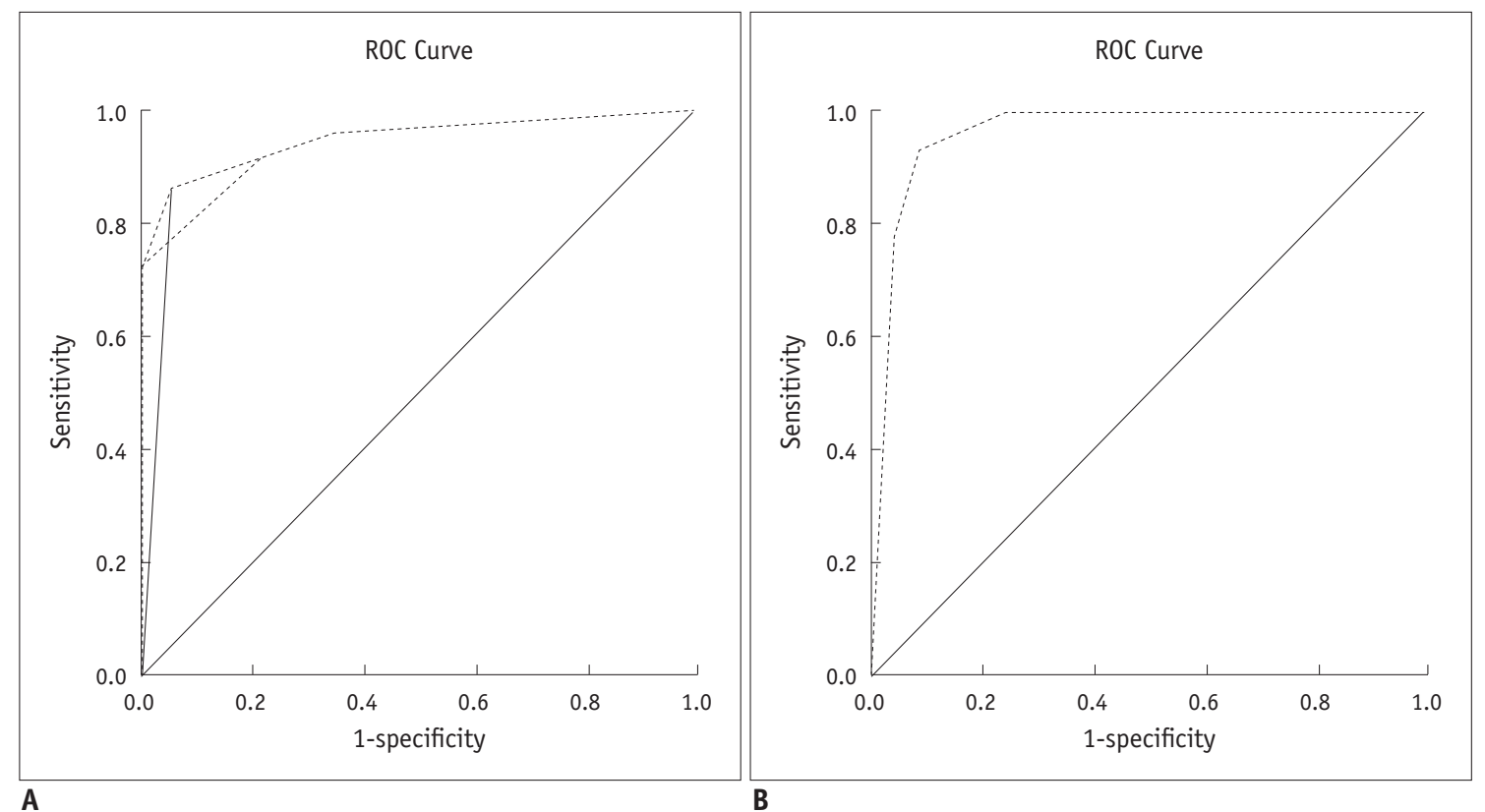

Fig. 4. Receiver-operating characteristic (ROC) analysis.

A. Receiver-operating characteristic curves for cut-off US criteria of malignancy. When nodules having 1, 2, or 3 or more malignant US features were considered malignant, Az values were 0.940 (dotted line), 0.950 (dashed line), and 0.931 (dotted and dashed line), respectively. B. Receiver-operating characteristic curve of US classification system when two or more malignant US features were considered as criteria of malignant US diagnosis (Az value: 0.950 , confidence interval: $0.914-0.986$ ). Sensitivity, specificity, positive and negative predictive values, and accuracy of US diagnosis were determined to be $86 \%, 95 \%, 91 \%, 92 \%$, and $92 \%$, respectively when malignant and suspicious for malignancy cases were collectively classified as malignant and benign, while probably benign and borderline were collectively classified as benign. 
Table 3. Diagnostic Indices for Individual US Feature of Malignant and Benign Thyroid Nodules

\begin{tabular}{llccccc}
\hline US Features & & Sensitivity (\%) & Specificity (\%) & PPV (\%) & NPV (\%) & Accuracy (\%) \\
\hline Malignant & Marked hypoechogenicity & $46 / 72(64)$ & $117 / 119(98)$ & $46 / 48(96)$ & $117 / 143(82)$ & $163 / 191(85)$ \\
& Marginal abnormality* & $50 / 72(69)$ & $117 / 119(98)$ & $50 / 52(96)$ & $117 / 139(84)$ & $167 / 191(87)$ \\
& Microcalcifications & $32 / 72(44)$ & $117 / 119(98)$ & $32 / 34(94)$ & $117 / 157(75)$ & $149 / 191(78)$ \\
& Taller-than-wide shape & $46 / 72(64)$ & $119 / 119(100)$ & $46 / 46(100)$ & $119 / 143(83)$ & $165 / 191(86)$ \\
& $\begin{array}{l}\text { Perithyroidal } \\
\text { lymphadenopathy** }\end{array}$ & $13 / 72(18)$ & $119 / 119(100)$ & $13 / 13(100)$ & $119 / 178(67)$ & $132 / 191(69)$ \\
Borderline*** & Hypoechogenicity & $13 / 119(11)$ & $56 / 72(78)$ & $13 / 29(45)$ & $56 / 162(35)$ & $69 / 191(36)$ \\
& Centrally predominant & $7 / 119(6)$ & $64 / 72(89)$ & $7 / 15(47)$ & $64 / 176(36)$ & $71 / 191(37)$ \\
& vascularity & $12 / 119(10)$ & $62 / 72(86)$ & $12 / 22(55)$ & $62 / 169(37)$ & $74 / 191(39)$ \\
\cline { 3 - 6 } Benign & Macrocalcifications & $96 / 119(81)$ & $66 / 72(92)$ & $96 / 102(94)$ & $66 / 89(74)$ & $162 / 191(85)$ \\
& Ovoid or flat shape & $48 / 119(40)$ & $66 / 72(92)$ & $48 / 54(89)$ & $66 / 137(48)$ & $114 / 191(60)$ \\
& Isoechogenicity & $72 / 119(61)$ & $53 / 72(74)$ & $72 / 91(79)$ & $53 / 100(53)$ & $125 / 191(65)$ \\
& Smooth margin & $75 / 119(63)$ & $70 / 72(97)$ & $75 / 77(97)$ & $70 / 114(61)$ & $145 / 191(76)$ \\
\hline
\end{tabular}

Note. - * Marginal abnormality with microlobulation, spiculated margin, or perithyroidal infiltration, ** Perithyroidal lymphadenopathy with intranodal microcalcifications or cystic component, ${ }^{* *}$ Diagnostic index of borderline US features when these were classified as benign. NPV = negative predictive value, $P P V=$ positive predictive value

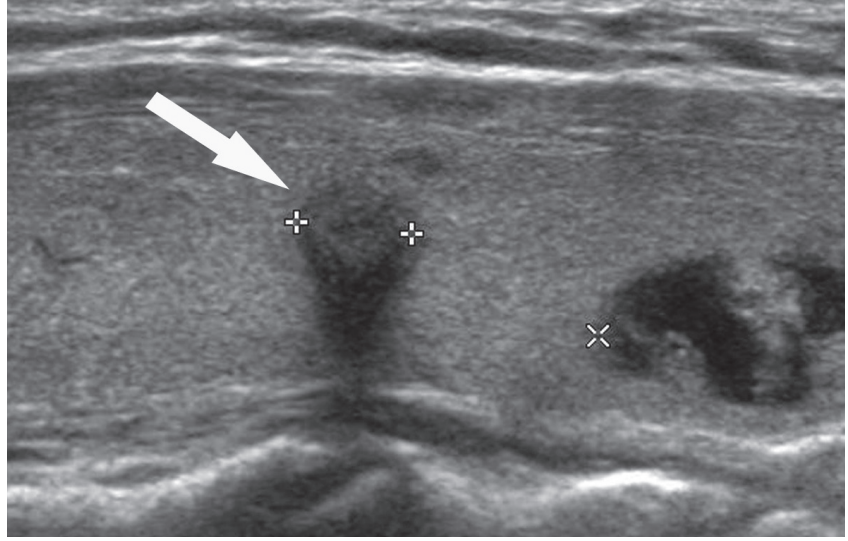

Fig. 5. Benign nodule designated as suspicious for malignancy as result of thyroid US in 52-year-old woman (false positive). Longitudinal US image of thyroid nodule in left mid-lobe shows hypoechogenicity, spiculated margin, and round shape (arrow); however, lesion was confirmed as spontaneously collapsed nodule of previous benign cystic thyroid nodule by consecutive US-fine needle aspirations and follow-up US.

were significant for ROC analysis (Fig. 4A). When nodules having two or more malignant US features were considered malignant, the $A z$ value was 0.950 , which is the highest among $\mathrm{Az}$ values. An ROC analysis revealed that the diagnostic accuracy of thyroid US using the present US classification system was very good $(A z=0.950)$ (Fig. 4B). In addition, diagnostic indices for individual US features of malignant and benign solid thyroid nodules are shown in Table 3.

All of the nodules designated as malignant upon thyroid US, were confirmed to be papillary thyroid carcinomas (PTCs) with the exception of one medullary thyroid carcinoma. Sixteen nodules designated as suspicious for malignancy included 10 PTCs, four pseudonodules related to thyroiditis, one benign lesion confirmed as a spontaneous collapse of a previously benign cystic nodule, and one nodular hyperplasia (Fig. 5). Out of the 82 nodules designated as benign, three PTCs and 79 benign nodules were identified, including six cases of nodular hyperplasia and one case of follicular adenoma, each confirmed by surgery (Fig. 6). Of the 18 nodules designated as probably benign, three PTCs were surgically confirmed. Pathological diagnoses in the borderline US category included three PTCs, one follicular thyroid carcinoma, five follicular adenomas, one Hurthle cell adenoma, and six cases of nodular hyperplasia. However, for the seven thyroid nodules designated as borderline, the patients did not undergo thyroid surgery due to benign cytological results after US-FNA.

\section{DISCUSSION}

High-resolution thyroid US is the most useful diagnostic tool for evaluating thyroid nodules. Numerous published studies report the diagnostic accuracy of thyroid US for thyroid nodules, and several US characteristics have been introduced as potential predictors of thyroid malignancy (1-19). However, there is a considerable overlap of the US characteristics between benign and malignant thyroid nodules in many reports (1-17). In addition, these studies 

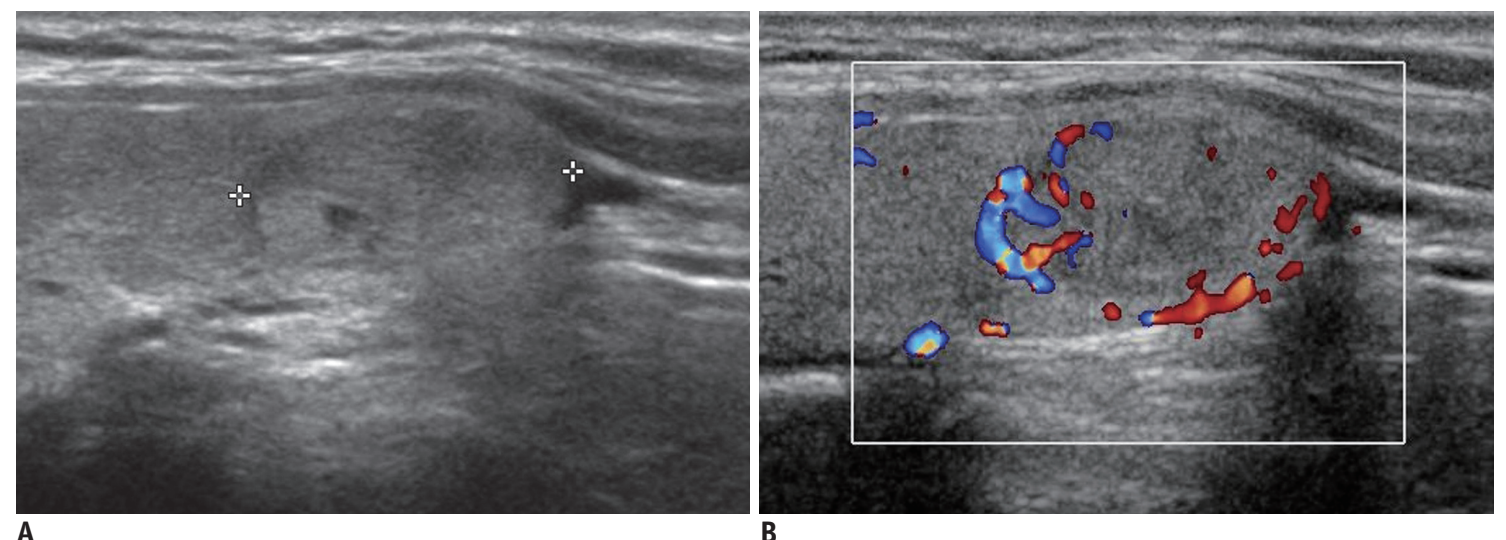

Fig. 6. Papillary thyroid carcinoma designated as benign from thyroid US in 18-year-old woman (false negative). Longitudinal US image of right thyroid nodule shows ovoid shape, isoechogenicity, smooth margin, and peripheral vascularity (A, B).

are either retrospective or do not include a systemized diagnostic classification for thyroid US. In particular, retrospective evaluations of thyroid images are limited due to being restricted to a pre-existing set of images.

We attempted to determine the diagnostic accuracy of a US classification system using 5 categories for solid thyroid nodules and real-time US. To our knowledge, no studies use different US diagnosis systems according to solid or cystic thyroid nodules. Recently, one of the authors reported the high accuracy of real-time US diagnosis for partially cystic thyroid nodules using a US classification system with 4 categories (15). Kim et al. (1) reported that the sensitivity of thyroid US is high for non-palpable thyroid nodules; they also reported that US findings for non-palpable thyroid nodules are different for benign and malignant lesions and can be helpful for differentiating between the abovementioned lesions. Compared with the study by Kim et al. (1), the diagnostic accuracy of thyroid US is greater in the present study. We believe that there are several reasons behind the high diagnostic accuracy of thyroid US in the present study. First, we used a US classification system with 5 categories. Second, we used real-time US and a relatively higher resolution US modality compared with the previous study (1). Finally, the present study describes the existence of lymphadenopathy with intranodal microcalcifications or cystic components, which are sonographic features of associated lymph nodes in favor of malignancy, as described in previous studies (17-19). Recently, Horvath et al. (4) have reported that an ultrasonography-based reporting system improved patient management and cost-effectiveness by helping avoid unnecessary FNA. However, the diagnostic indices in their study were similar to or lower than those of our study, though they used the clinical information of patients in the determination of US classification. In addition, we considered lymph nodes associated with intranodal microcalcifications or cystic components in the perithyroidal region to be malignant criteria when there was no mass within the visceral space, with the exception of the thyroid upon US examination. However, further study is required to evaluate the significance of the lymph nodes associated with intranodal microcalcifications or cystic components in diagnosis of thyroid nodules.

We classified US diagnoses of solid thyroid nodules into 5 categories. Because all nodules assigned to the malignant US category upon thyroid US were confirmed as PTCs, we presume that the possibility of a benign lesion could be excluded if a thyroid nodule had two or more US features suggestive of malignancy as observed in the thyroid US. In the case of the suspicious for malignancy category, the incidence of malignancy is obviously high; however, the possibility of a benign lesion should be considered. The borderline US category includes a considerable number of benign and malignant nodules, although benign nodules were predominant among them. If a thyroid nodule is classified into the borderline category in the thyroid US, we recommend one or more trials of consecutive US-FNAs.

Three borderline US features were included in the present study. The specificity of hypoechogenicity for the diagnosis of malignant thyroid nodules remains uncertain, whereas marked hypoechogenicity is regarded as a significantly specific criterion $(1-3,5-8,11)$. The diagnostic accuracy of color Doppler US remains controversial with respect to the differentiation of benign and malignant thyroid nodules (5-7). Frates et al. (7) observed centrally predominant vascularity in a higher percentage of malignant nodules than benign nodules ( $42 \%$ and $14 \%$ respectively). In 
addition, controversy remains over the likelihood of malignancy according to the presence, pattern, or shape of macrocalcifications in thyroid nodules $(9,20-22)$, as some investigators report that interrupted eggshell calcifications on thyroid nodules suggest malignancy (2326). In this study, the incidence of hypoechogenicity, centrally predominant vascularity, and macrocalcifications were $28 \%$ (20 of 72), $11 \%$ ( 8 of 72 ), and 15\% (11 of 72) for malignant nodules, and $9 \%$ (11 of 119), $6 \%$ (7 of 119), and $9 \%$ (11 of 119) for benign nodules, respectively. This result is consistent with our assumption that borderline US features are indeterminate for malignancy. However, further study is required to evaluate the significance of these borderline US features.

There are several limitations to this study. First, only one dominant thyroid nodule for each patient was selected. This, in addition to the high incidence of thyroid malignancy, might have resulted in a bias. Second, 81 nodules assigned to benign US categories and benign cytology after US-FNA only underwent a follow-up thyroid US after at least 12 months without additional US-FNA or thyroid surgery. However, they showed no suspicious US features or significant increase in nodular size on followup US. However, US follow-up intervals after 12 months might to be too short to confirm the benignity of the thyroid nodules. Third, the incidence rate of PTCs for thyroid malignancy was $97 \%$ (70 of 72). Finally, all US diagnoses were determined by a single radiologist. As a result, a multicenter study is required.

In summary, the diagnostic accuracy of thyroid US for solid thyroid nodules is good when the 5-category US classification system is applied.

\section{REFERENCES}

1. Kim EK, Park CS, Chung WY, Oh KK, Kim DI, Lee JT, et al. New sonographic criteria for recommending fine-needle aspiration biopsy of nonpalpable solid nodules of the thyroid. AJR Am J Roentgenol 2002;178:687-691

2. Cappelli C, Castellano M, Pirola I, Cumetti D, Agosti B, Gandossi $\mathrm{E}$, et al. The predictive value of ultrasound findings in the management of thyroid nodules. OJM 2007;100:29-35

3. Salmaslioglu A, Erbil Y, Dural C, Issever H, Kapran Y, Ozarmagan $S$, et al. Predictive value of sonographic features in preoperative evaluation of malignant thyroid nodules in a multinodular goiter. World J Surg 2008;32:1948-1954

4. Horvath E, Majlis S, Rossi R, Franco C, Niedmann JP, Castro $A$, et al. An ultrasonogram reporting system for thyroid nodules stratifying cancer risk for clinical management. J Clin
Endocrinol Metab 2009;94:1748-1751

5. Papini E, Guglielmi R, Bianchini A, Crescenzi A, Taccogna $S$, Nardi $F$, et al. Risk of malignancy in nonpalpable thyroid nodules: predictive value of ultrasound and color-Doppler features. J Clin Endocrinol Metab 2002;87:1941-1946

6. Iannuccilli JD, Cronan JJ, Monchik JM. Risk for malignancy of thyroid nodules as assessed by sonographic criteria: the need for biopsy. J Ultrasound Med 2004;23:1455-1464

7. Frates MC, Benson CB, Doubilet PM, Cibas ES, Marqusee E. Can color Doppler sonography aid in the prediction of malignancy of thyroid nodules? J Ultrasound Med 2003;22:127-131; quiz 132-124

8. Shimura H, Haraguchi K, Hiejima Y, Fukunari N, Fujimoto $Y$, Katagiri $M$, et al. Distinct diagnostic criteria for ultrasonographic examination of papillary thyroid carcinoma: a multicenter study. Thyroid 2005;15:251-258

9. Moon WJ, Jung SL, Lee JH, Na DG, Baek JH, Lee YH, et al. Benign and malignant thyroid nodules: US differentiation-multicenter retrospective study. Radiology 2008;247:762-770

10. Tae HJ, Lim DJ, Baek KH, Park WC, Lee YS, Choi JE, et al. Diagnostic value of ultrasonography to distinguish between benign and malignant lesions in the management of thyroid nodules. Thyroid 2007;17:461-466

11. Katz JF, Kane RA, Reyes J, Clarke MP, Hill TC. Thyroid nodules: sonographic-pathologic correlation. Radiology 1984;151:741745

12. Brander A, Viikinkoski P, Nickels J, Kivisaari L. Thyroid gland: US screening in a random adult population. Radiology 1991;181:683-687

13. Wienke JR, Chong WK, Fielding JR, Zou KH, Mittelstaedt CA. Sonographic features of benign thyroid nodules: interobserver reliability and overlap with malignancy. J Ultrasound Med 2003;22:1027-1031

14. Brkljacic B, Cuk V, Tomic-Brzac H, Bence-Zigman Z, DelicBrkljacic D, Drinkovic I. Ultrasonic evaluation of benign and malignant nodules in echographically multinodular thyroids. J Clin Ultrasound 1994;22:71-76

15. Kim DW, Lee EJ, In HS, Kim SJ. Sonographic differentiation of partially cystic thyroid nodules: a prospective study. AJNR Am J Neuroradiol 2010;31:1961-1966

16. Lee MJ, Kim EK, Kwak JY, Kim MJ. Partially cystic thyroid nodules on ultrasound: probability of malignancy and sonographic differentiation. Thyroid 2009;19:341-346

17. Frates MC, Benson CB, Charboneau JW, Cibas ES, Clark $\mathrm{OH}_{\text {, }}$ Coleman BG, et al. Management of thyroid nodules detected at US: Society of Radiologists in Ultrasound consensus conference statement. Radiology 2005;237:794-800

18. Cooper DS, Doherty GM, Haugen BR, Kloos RT, Lee SL, Mandel SJ, et al. Revised American Thyroid Association management guidelines for patients with thyroid nodules and differentiated thyroid cancer. Thyroid 2009;19:1167-1214

19. Moon WJ, Baek JH, Jung SL, Kim DW, Kim EK, Kim JY, et al. Ultrasonography and the ultrasound-based management of thyroid nodules: consensus statement and recommendations. Korean J Radiol 2011;12:1-14 
20. Kessler A, Rappaport Y, Blank A, Marmor S, Weiss J, Graif M. Cystic appearance of cervical lymph nodes is characteristic of metastatic papillary thyroid carcinoma. J Clin Ultrasound 2003;31:21-25

21. Ying M, Ahuja A, Metreweli C. Diagnostic accuracy of sonographic criteria for evaluation of cervical lymphadenopathy. J Ultrasound Med 1998;17:437-445

22. Kakkos SK, Scopa CD, Chalmoukis AK, Karachalios DA, Spiliotis JD, Harkoftakis JG, et al. Relative risk of cancer in sonographically detected thyroid nodules with calcifications. J Clin Ultrasound 2000;28:347-352

23. Kwak MS, Baek JH, Kim YS, Jeong HJ. Patterns and
Significance of Peripheral Calcifications of Thyroid Tumors seen on Ultrasound. J Korean Radiol Soc 2005;53:401-405

24. Wang N, Xu Y, Ge C, Guo R, Guo K. Association of sonographically detected calcification with thyroid carcinoma. Head Neck 2006;28:1077-1083

25. Kim BM, Kim MJ, Kim EK, Kwak JY, Hong SW, Son EJ, et al. Sonographic differentiation of thyroid nodules with eggshell calcifications. J Ultrasound Med 2008;27:1425-1430

26. Park M, Shin JH, Han BK, Ko EY, Hwang HS, Kang SS, et al. Sonography of thyroid nodules with peripheral calcifications. $\mathrm{J}$ Clin Ultrasound 2009;37:324-328 